\title{
End-User Software Engineering: Toward a Future Beyond the Silos
}

\author{
Margaret Burnett \\ School of EECS, Oregon State University \\ Corvallis, OR 97331 USA \\ burnett@eecs.oregonstate.edu
}

\begin{abstract}
This paper summarizes the keynote address on the future of end-user software engineering. We believe the future that we envision has implications for not only end-user software engineering, but also for "classic" software engineering.
\end{abstract}

Keywords: End-user software engineering (EUSE), end-user programming, end-user development.

\section{From End-User Programming to End-User Software Engineering}

End-user programming has become pervasive in our society [3], with end users programming simulations, courseware, spreadsheets, macros, mashups, and more $[1,2]$. In this talk, we will consider what happens when we add to end-user programming environments consideration of the software lifecycle beyond the "coding" phase. Considering other phases is necessary, because there is ample evidence that the programs end users create are filled with errors [1]. To help address this problem, we have been working on a software "engineering" methodology designed specifically for end users.

Because the user may have no expertise or even interest in software engineering, we do not propose to transform end users into engineers. Rather, most end-user software engineering research strives to tightly integrate elements of design, implementation, component integration, debugging, testing, and maintenance, supported behind the scenes by analysis and inferential reasoning, to help the user reason about the dependability of their software as they work with it, in a manner that respects the user's problem-solving directions to an extent unprecedented in existing software development environments [2].

In this talk, we briefly describe the present state of end-user software engineering, and then focus on challenges in moving forward - specifically, the challenges of avoiding becoming over-siloed. We then show that focusing on the in-the-moment intents of end-user developers can be used to derive a number of promising directions forward for end-user software engineering researchers, and how theories can help us further de-silo future end-user software engineering research. Finally, we discuss how overcoming challenges for the future of end-user 
software engineering may also bring direct benefits to the future of "classic" software engineering.

Biography: Margaret Burnett's research is in human issues of software development, which lies in the intersection of HCI and software engineering. Her current research focuses on end-user programming, end-user software engineering, information foraging theory as applied to programming, and gender issues in those contexts. End-user software engineering is the first research area to rigorously consider the problem of dependability in end-user programming, and Burnett led in founding this research area. Her team's "WYSIWYT" systematic testing approach for end-user programmers initiated the groundwork, and in 2003, she co-founded and became Project Director of the EUSES Consortium. Under her leadership, this collaboration grew to 10 institutions whose contributions have helped ordinary end users achieve up to 10 times greater effectiveness at guarding against software defects, receiving wide recognition for technical quality (11 Best Paper recognitions for the project). Burnett's awards for her own work include several Best Paper recognitions, IBM's International Faculty Award, and the NSF Young Investigator Award. She was recently honored with her university's Excellence in Graduate Mentoring Award, College of Engineering's Research Award, College of Engineering's Research Collaboration Award, and the Elizabeth P. Ritchie Distinguished Professor Award. She also serves on a variety of HCI and Software Engineering conference program committees, and currently co-chairs the Academic Alliance of the National Center for Women \& Information Technology (NCWIT).

Acknowledgments. This talk is an expanded version of a talk given at the ICSE'14 "Future of Software Engineering" track; both are based on an invited paper co-authored by Burnett and Myers [1]. We'd like to acknowledge the many researchers whose pioneering works have helped establish the area of end-user software engineering. We also thank the students and collaborators who contributed to our own work in end-user software engineering. This paper was supported in part by the National Science Foundation under grants 1240957, 1314384, 1302113. Any opinions, findings and conclusions or recommendations expressed in this material are those of the author(s) and do not necessarily reflect those of the National Science Foundation.

\section{References}

1. Burnett, M., Myers, B.: Future of End-User Software Engineering: Beyond the Silos. In: ACM/IEEE International Conference on Software Engineering: Future of Software Engineering Track (ICSE Companion Proceedings), pp. 201-211. ACM Press (2014)

2. Ko, A., Abraham, R., Beckwith, L., Blackwell, A., Burnett, M., Erwig, M., Scaffidi, C., Lawrance, J., Lieberman, H., Myers, B., Rosson, M., Rothermel, G., Shaw, M., Wiedenbeck, S.: The State of the Art in End-User Software Engineering. ACM Computing Surveys 43(3), Article 21, 44 pages (2011)

3. Scaffidi, C., Shaw, M., Myers, B.: Estimating the Numbers of End Users and End User Programmers. In: IEEE Symposium on Visual Languages and Human-Centric Computing, pp. 207-214. IEEE (2005) 